\title{
Self-rated cognitive functions following chemotherapy in patients with breast cancer: a 6-month prospective study
}

This article was published in the following Dove Press journal:

Neuropsychiatric Disease and Treatment

3 October 2017

Number of times this article has been viewed

\author{
Ryosuke Kitahata' \\ Shinichiro Nakajima ${ }^{1-4}$ \\ Hiroyuki Uchida ${ }^{1,3}$ \\ Tetsu Hayashida ${ }^{5}$ \\ Maiko Takahashi ${ }^{5}$ \\ Shintaro Nio' \\ Jinichi Hirano' \\ Maki Nagaoka' \\ Takefumi Suzuki' \\ Hiromitsu Jinno ${ }^{6}$ \\ Yuko Kitagawa ${ }^{5}$ \\ Masaru Mimura' \\ 'Psychopharmacology Research \\ Program, Department of \\ Neuropsychiatry, School of Medicine, \\ Keio University, Tokyo, Japan; \\ ${ }^{2}$ Multimodal Imaging Group - Research \\ Imaging Centre, ${ }^{3}$ Geriatric Mental \\ Health Division, Centre for Addiction \\ and Mental Health, ${ }^{4}$ Department of \\ Psychiatry, University of Toronto, \\ Toronto, Canada; ${ }^{5}$ Department of \\ Surgery, School of Medicine, Keio \\ University, ${ }^{6}$ Department of Surgery, \\ School of Medicine, Teikyo University, \\ Tokyo, Japan
}

Purpose: The purpose of the study was to evaluate subjective (self-rated), family-rated, and objective (researcher-rated) cognitive functions in patients with breast cancer after chemotherapy.

Method: We conducted a prospective study to trace self-rated cognitive functions in 30 patients with breast cancer at the completion of chemotherapy (T0) and 6 months later (T1). Subjective cognitive functions were assessed with Cognitive Failures Questionnaire (CFQ), Dysexecutive Questionnaire (DEX-S), and Everyday Memory Checklist (EMC-S) for attention, executive function, and episodic memory, respectively. Their family members also completed DEX-I and EMC-I for executive function and episodic memory, respectively. We also examined objective cognitive functions. Self-rated cognitive functions were compared with the normative data. They were compared between $\mathrm{T} 0$ and $\mathrm{T} 1$. We calculated correlation coefficients between self-rated and other cognitive functions.

Results: At T0, 6 (20.0\%) and 2 (6.7\%) participants showed higher DEX-S and EMC-S scores than the normative data, respectively, while no participant had abnormal CFQ scores. At T1, DEX-S and EMC-S scores were normalized in 3 (50.0\%) and 2 (100.0\%) participants, respectively. No participant showed increases in CFQ scores. No changes were found in objective cognitive functions from T0 to T1. DEX-S and DEX-I or EMC-S and EMC-I scores were correlated at both $\mathrm{T} 0$ and $\mathrm{T} 1$, which did not survive multiple corrections. There was no association between subjective and objective cognitive functions.

Conclusion: Impairments in subjective cognition may be transient after chemotherapy in patients with breast cancer. Furthermore, patients and their families appear to share similar prospects on their cognitive functions.

Keywords: breast cancer, chemotherapy, subjective cognitive functions

\section{Introduction}

Breast cancer is the most common form of cancer among women. ${ }^{1}$ Cancer-related cognitive impairment has gathered attention since cognitive impairment can persist for months to years after treatment, and even subtle impairments can have profound consequences upon quality of life (QOL) and social functioning. ${ }^{2}$ Based on previous longitudinal studies, ${ }^{3-7}$ Wefel et al have estimated that up to $75 \%$, and up to $60 \%$ of cancer patients show cognitive impairment with mild-to-moderate severity during treatment and after the completion of cancer treatment, respectively. ${ }^{2}$ On the other hand, cognitive impairment has been reported to be already present in approximately $40 \%$ of patients even before the start of any treatment, which complicates the story. ${ }^{2}$
Correspondence: Shinichiro Nakajima Psychopharmacology Research Program, Department of Neuropsychiatry, School of Medicine, Keio University, 35 Shinanomachi, Shinjuku-ku, Tokyo, 160-8582, Japan

Tel +8I 33353 I2II (ext 62454)

Fax +81353790187

Email shinichiro_nakajima@hotmail.com 
The subjective component of cognitive impairment following chemotherapy is also important, given that selfreported cognitive symptoms appear to be strongly associated with poor psychological well-being in these patients. ${ }^{2}$ Several studies have prospectively examined perceived cognitive functions using standardized questionnaires in patients with breast cancer. ${ }^{8-15}$ However, the findings have been inconsistent with respect to reported differences between patients with breast cancer and healthy controls ${ }^{9,12}$ or between patients with breast cancer and those with benign breast tumor. ${ }^{10,15}$ Previous findings on prospective (pretreatment and posttreatment) changes within patients with breast cancer have also been mixed. ${ }^{9-15}$ Moreover, the outcomes for subjective cognitions thus far are limited to attention ${ }^{9,12}$ and global cognition. ${ }^{11}$ Furthermore, cognitive functions as assessed by family members have not been reported in the literature to our knowledge. Since family members live closely with patients, they may well be positioned to notice changes in patients in an objective manner, which has a critical significance on its own right. Thus, research is urgently needed to examine subjective cognitive functioning in patients with breast cancer, assessed by patients and their family, using standardized batteries covering various cognitive domains.

Given these backgrounds, we conducted a prospective study to evaluate self-rated and family-rated cognitive functions such as attention, executive function, and episodic memory in patients with breast cancer for 6 months after the completion of adjuvant/neoadjuvant chemotherapy. Other outcomes included objective cognitive functions, depressive/ anxious symptoms, fatigue, motivation, and QOL. Subjective cognitive functions within 4 weeks after chemotherapy (T0) and 6 months after T0 (T1) were compared with the normative data. Subjective cognitive functions were also prospectively compared between T0 and T1. Furthermore, to shed light on the mechanistic insight for cognitive difficulties, we explored the relationship between subjective cognitive functions and the levels of tumor necrosis factor- $\alpha$ (TNF- $\alpha$ ) and hemoglobin since inflammation ${ }^{16}$ and anemia ${ }^{17}$ have been implicated in cognitive impairment, respectively.

\section{Methods}

\section{Study design}

This 6-month open-label prospective study was conducted at Keio University Hospital in Tokyo, Japan, from April 2012 to August 2015. Thirty patients with breast cancer who planned to undergo adjuvant/neoadjuvant chemotherapy were included. Participants were excluded if they had a history of brain radiation therapy, a serious medical condition such as another malignancy, a history of serious psychiatric diseases, or substance use disorders. This study was approved by the Institutional Ethics Board of Keio University Hospital. All participants provided written informed consent after a full description of the study at Keio University Hospital. Assessments were performed within 4 weeks after the completion of chemotherapy (T0) and 6 months after T0 (T1).

\section{Outcome measures}

The following information was collected at T0: age, sex, current medications, age at onset, stage of breast cancer, treatment history of breast cancer, history of alcohol drinking and smoking, educational background, and family history.

All of the following outcomes were examined at $\mathrm{T} 0$ and T1. Subjective cognitive functions were assessed with the Cognitive Failures Questionnaire (CFQ), ${ }^{18}$ Dysexecutive Questionnaire (DEX), ${ }^{19}$ and Everyday Memory Checklist $(\mathrm{EMC})^{20}$ for attention, executive function, and episodic memory, respectively, since chemotherapy can induce mild impairments in these objective cognitions in patients with breast cancer. ${ }^{21}$ The DEX and EMC were administered to both participants and their family members (self-rated DEX [DEX-S] and independent-rater-rated DEX [DEX-I], self-rated EMC [EMC-S] and independent-rater-rated EMC [EMC-I], respectively). The primary outcome measures for this study included the DEX-S, EMC-S, and CFQ scores. For these rating scales, higher scores represent greater impairment in subjective cognitive functions.

Objective cognitive functions were assessed using the Behavioural Assessment of the Dysexecutive Syndrome $(B A D S)^{19}$ for executive function, Rivermead Behavioural Memory Test (RBMT) ${ }^{22}$ for episodic memory, and LetterNumber Sequencing (LNS) ${ }^{23}$ and Visual Memory Span forward and backward (VMS-f \& -b) ${ }^{24}$ for working memory. For these rating scales, higher scores represent better objective cognitive functions.

Other rating scales included the Montgomery-Åsberg Depression Rating Scale (MADRS), ${ }^{25}$ Hamilton Anxiety Rating Scale (HAMA), ${ }^{26}$ Brief Fatigue Inventory (BFI), ${ }^{27}$ Clinical Assessment for Spontaneity (CAS) ${ }^{28}$ and Functional Assessment of Cancer Therapy-Breast (FACT-B), ${ }^{29}$ which assessed depression, anxiety, fatigue, motivation, and QOL, respectively.

\section{Blood sampling}

Venous blood was collected at $\mathrm{T} 0$ and $\mathrm{T} 1$ to measure levels of TNF- $\alpha$ and hemoglobin. Plasma concentrations of 
TNF- $\alpha$ were detected by sandwich ELISA according to the manufacturer's instructions (RPN5967; GE Healthcare UK, Buckinghamshire, UK) with a detection limit of $2.5 \mathrm{pg} / \mathrm{mL}$. Plasma concentrations of hemoglobin were measured by the SLS-Hb method (LSI Medience, Tokyo, Japan) with a detection limit of $0.10 \mathrm{~g} / \mathrm{dL}$.

\section{Statistical analyses}

Means and standard deviations (SD) were calculated for clinicodemographic and cognitive data at T0 and T1. The CFQ, DEX, EMC, BADS, RBMT, LNS, and VMS scores were compared with the normative data by converting each to a $z$-score at T0 and T1. If the $z$-score was higher than 1.5 , the corresponding cognitive function was defined as impaired based on the previous literature. ${ }^{2}$ The CFQ, DEX, EMC, BADS, RBMT, LNS, VMS, MADRS, HAMA, BFI, $\mathrm{CAS}$, and FACT-B scores and levels of TNF- $\alpha$ and hemoglobin were compared between $\mathrm{T} 0$ and $\mathrm{T} 1$ using Wilcoxon signed-rank tests with the last observation carried forward (LOCF) method. These variables at T0 and T1 and changes in these variables from $\mathrm{T} 0$ to $\mathrm{T} 1$ were also compared between those who received radiotherapy/hormone therapy and those who did not using Mann-Whitney $U$-tests with the LOCF method to explore the influences of these treatments on cognitive functions.

We explored Spearman's rank correlation coefficients between the CFQ, DEX-S, and EMC-S scores at T0 and age, duration of illness, duration of education and the CFQ, DEX, EMC, BADS, RBMT, LNS, VMS, MADRS, HAMA, BFI, CAS, and FACT-B scores, and levels of TNF- $\alpha$ and hemoglobin at T0. Spearman's rank correlation coefficients were also used to examine the relationship between changes from $\mathrm{T} 0$ to $\mathrm{T} 1$ in the CFQ, DEX-S, and EMC-S scores and changes from $\mathrm{T} 0$ to $\mathrm{T} 1$ in the CFQ, DEX, EMC, BADS, RBMT, LNS, VMS, MADRS, HAMA, BFI, CAS, and FACT-B scores, and levels of TNF- $\alpha$ and hemoglobin.

Owing to the number of comparisons, significance levels were set at a $P$-value of $<0.05 /$ number of performed analyses and all tests were two-tailed. We considered multiple corrections separately for primary (ie, CFQ, DEX-S, and EMC-S scores) and secondary outcome measures. Thus, we considered that a significant $P$-value is less than 0.016 $(=0.05 / 3)$ and $0.0038(=0.05 /(16-3))$ for the comparisons in primary and secondary outcomes, respectively, between T0 and T1. A $P$-value is also considered significant if it is less than $0.016(=0.05 / 3)$ and $0.0011(=0.05 /(3 \times 16-3))$ for the correlation analyses between primary outcomes and the other correlation analyses, respectively. All statistical analyses were conducted using IBM SPSS Statistics version 20 (IBM Corporation, Armonk, NY, USA). Continuous and categorical variables were described as the mean $\pm \mathrm{SD}$ and number $(\%)$, respectively.

\section{Results \\ Participants}

Sociodemographic and clinical characteristics at T0 are summarized in Table 1. A total of 30 participants were enrolled in this study. Eight (26.7\%) and 11 (36.7\%) participants had stage IIa and IIb cancer, respectively, at T0. Twenty-seven $(90.0 \%)$ and $3(10.0 \%)$ participants had completed neoadjuvant therapy and adjuvant therapy at $\mathrm{T} 0$, respectively. Those who had received neoadjuvant therapy underwent surgery 12.0 \pm 7.8 days after T0. Thirteen (48.1\%) participants underwent radiotherapy $67.8 \pm 36.6$ days after T0 with treatment duration of $36.4 \pm 2.4$ days. Eighteen $(60.0 \%)$ participants received hormone therapy during $\mathrm{T} 0$ and $\mathrm{T} 1$, while $9(30.0 \%)$ participants did not among 27 patients who had not received this treatment at T0. Three $(10.0 \%)$ participants dropped out between T0 and T1 due to feeling sick $(n=2)$ and difficulty in managing arrangements $(n=1)$.

\section{Subjective cognitive functions}

Table 2 depicts the cognitive functions and other psychological symptoms at T0 and T1. At T0, 6 (20.0\%) and $5(16.7 \%)$ participants showed higher DEX-S and DEX-I scores, respectively, than the normal standard. Two $(6.7 \%)$

Table I Clinicodemographic characteristics at T0 and TI

\begin{tabular}{|c|c|c|}
\hline & T0 & TI \\
\hline Number of subjects (females) & $30(30)$ & $27(27)$ \\
\hline Age, mean $\pm S D$, years & $54.0 \pm 10.0$ & $54.0 \pm 10.2$ \\
\hline Disease duration, mean $\pm S D$, days & $478.0 \pm 1,093.1$ & \\
\hline \multicolumn{3}{|l|}{ Stage, n (\%) } \\
\hline 1 & $2(6.7)$ & $2(7.4)$ \\
\hline II & $20(66.7)$ & $17(63.0)$ \\
\hline III & $7(23.3)$ & $7(25.9)$ \\
\hline IV & I (3.3) & I (3.7) \\
\hline \multicolumn{3}{|l|}{ Method of chemotherapy, n (\%) } \\
\hline Neoadjuvant chemotherapy & $27(90.0)$ & $24(88.9)$ \\
\hline DTX + TS-I and FEC & $20(66.7)$ & $18(66.7)$ \\
\hline $\mathrm{HER}+\mathrm{PTX}$ & $7(23.3)$ & $6(22.2)$ \\
\hline Adjuvant therapy & $3(10.0)$ & $3(11.1)$ \\
\hline DTX + TS-I and FEC & $3(10.0)$ & $3(I I . I)$ \\
\hline HER + PTX & $0(0.0)$ & $0(0.0)$ \\
\hline Hormone therapy, n (\%) & $3(10.0)$ & $21(77.8)$ \\
\hline Radiotherapy (partial irradiation), n (\%) & $0(0.0)$ & $13(48.1)$ \\
\hline Duration of education, mean $\pm \mathrm{SD}$, years & $13.7 \pm 1.7$ & $13.9 \pm 3.0$ \\
\hline
\end{tabular}

Abbreviations:DTX, docetaxel;FEC,5-fluorouracil+epirubicin+cyclophosphamide; HER, Herceptin ${ }^{\circledR}$ (trastuzumab); PTX, paclitaxel. 
Table 2 Comparisons of cognitive functions and other outcomes at T0 and TI

\begin{tabular}{|c|c|c|c|}
\hline Outcome measures & T0 & TI & Statistics \\
\hline DEX-S total scores & $11.9 \pm 9.3$ & $9.7 \pm 7.6$ & $z=-2, P=0.04$ \\
\hline DEX-I total scores & $8.6 \pm 10.2$ & $7.2 \pm 9.1$ & $z=-0.9, P=0.39$ \\
\hline BADS total scores & $18 \pm 4$ & $18.6 \pm 3.5$ & $z=-1.4, P=0.18$ \\
\hline EMC-S total scores & $7.6 \pm 5.6$ & $7.2 \pm 4.3$ & $z=-0.4, P=0.72$ \\
\hline EMC-I total scores & $4.5 \pm 5$ & $3.5 \pm 3.8$ & $z=-1.6, P=0.1$ \\
\hline RBMT total scores & $21.9 \pm 2.3$ & $22 \pm 1.7$ & $z=0, P=0.97$ \\
\hline CFQ total scores & $27.6 \pm 10.3$ & $26.9 \pm 10.1$ & $z=-0.6, P=0.57$ \\
\hline LNS scores & $13.3 \pm 2.5$ & $13.7 \pm 2.8$ & $z=-I . I, P=0.28$ \\
\hline VMS forward scores & $10.2 \pm 1.9$ & $10.2 \pm 1.6$ & $z=-0.3, P=0.79$ \\
\hline VMS backward scores & $9.4 \pm 1.5$ & $9.3 \pm 1.7$ & $z=-0.5, P=0.6$ \\
\hline CAS scores & $0.3 \pm 0.7$ & $0.1 \pm 0.3$ & $z=-1.7, P=0.1$ \\
\hline MADRS total scores & $3.8 \pm 2.8$ & $4 \pm 2.9$ & $z=-0.3, P=0.79$ \\
\hline HAM-A total scores & $6.5 \pm 4.3$ & $6.4 \pm 4.6$ & $z=-0.2, P=0.82$ \\
\hline BFI scores & $21.4 \pm 18.5$ & $15.7 \pm 16$ & $z=-1.6, P=0.12$ \\
\hline FACT-B total scores & $97.2 \pm 14.8$ & $103.0 \pm 17.1$ & $z=-2.3, P=0.02$ \\
\hline Hb levels & $11.6 \pm 0.9$ & $12.6 \pm I .1$ & $z=-3.9, P<0.0001$ \\
\hline TNF- $\alpha$ levels & $35.9 \pm 122.2$ & $18 \pm 33.9$ & $z=-1.9, P=0.06$ \\
\hline
\end{tabular}

Notes: We considered multiple corrections separately for primary (ie, CFQ, DEX-S, and EMC-S scores) and secondary outcome measures. Thus, we considered that a significant $P$-value is less than $0.016(=0.05 / 3)$ and $0.0038(=0.05 /[16-3])$ for the comparisons in primary and secondary outcomes, respectively, between T0 and TI. Bold characters represent statistically significant results. Data presented as mean \pm SD.

Abbreviations: BADS, Behavioural Assessment of the Dysexecutive Syndrome; BFI, Brief Fatigue Inventory; CAS, Clinical Assessment for Spontaneity; CFQ, Cognitive Failures Questionnaire; DEX-I, independent-rater-rated Dysexecutive Questionnaire; DEX-S, self-rated Dysexecutive Questionnaire; EMC-I, independent-rater-rated Everyday Memory Checklist; EMC-S, self-rated Everyday Memory Checklist; FACT-B, Functional Assessment of Cancer Therapy-Breast; HAM-A, Hamilton Anxiety Rating Scale; Hb, hemoglobin; LNS, Letter-Number Sequencing; MADRS, Montgomery-Åsberg Depression Rating Scale; RBMT, Rivermead Behavioural Memory Test; TNF- $\alpha$, tumor necrosis factor- $\alpha$; VMS, Visual Memory Span.

and $2(6.7 \%)$ participants showed higher EMC-S and EMC-I scores, respectively, than the normal standard. No participant had abnormal CFQ scores.

At T1, the DEX-S and DEX-I scores were decreased to a normal reference range in $3(3 / 6=50.0 \%)$ and $3(3 / 5=60.0 \%)$ participants, respectively. No participant showed increases in either of these scores. The EMC-S and EMC-I scores were decreased to a normal reference range in $2(2 / 2=100.0 \%)$ and $2(2 / 2=100.0 \%)$ participants, respectively, at T1. No participant showed increases in CFQ scores.

\section{Objective cognitive functions}

At T0, $5(16.7 \%)$ and $2(6.7 \%)$ participants showed lower BADS and RBMT scores, respectively, than the normal standard. There was no participant with abnormal LNS, VMS-f, and VMS-b scores.

At $\mathrm{T} 1$, the BADS and RBMT scores were normalized in $2(40.0 \%)$ and $1(50.0 \%)$ participants, respectively. One $(20.0 \%)$ participant dropped out among those who showed lower BADS scores at T0. No participant showed decreases in the BADS and RBMT scores. A decrease in LNS scores was observed in 1 (3.3\%) participant, who had lower scores than the normal standard. No participant showed decreases in the VMS-f and VMS-b scores.

\section{Correlations of self-rated cognitive functions and other clinicodemographic characteristics}

Correlational coefficients are shown in Tables 3 and 4. At T0, the DEX-S scores were related to the DEX-I, EMC-S, EMC-I, CFQ, and TNF- $\alpha$ scores. The EMC-S scores were associated with the DEX-S, DEX-I, EMC-I, CFQ, and BFI scores. The CFQ scores were associated with the DEX-S, EMC-S, and CAS scores (Table 3). After correction for multiple comparisons, the DEX-S, EMC-S, and CFQ scores were related to the EMC-S, DEX-S and CFQ, and EMC-S scores, respectively.

In terms of changes from $\mathrm{T} 0$ to $\mathrm{T} 1$, changes in the DEX-S scores were associated with changes in the FACT-B scores. Changes in the EMC-S scores were associated with changes in the CFQ and BFI scores. Changes in the CFQ scores were related to changes in the EMC-S and FACT-B scores and TNF- $\alpha$ levels (Table 4). However, these correlations did not survive correction for multiple comparisons.

\section{Impact of radiotherapy/hormone therapy on cognitive functions}

No significant differences were found in cognitive functions at $\mathrm{T} 0$ and $\mathrm{T} 1$, and changes in cognitive functions from 
Table 3 Correlations among self-rated cognitive functions and other clinicodemographic characteristics at TO

\begin{tabular}{|c|c|c|c|c|c|c|}
\hline \multirow[t]{3}{*}{ T0 } & \multicolumn{6}{|l|}{ TO } \\
\hline & \multicolumn{2}{|c|}{ DEX-S } & \multicolumn{2}{|c|}{ EMC-S } & \multicolumn{2}{|l|}{ CFQ } \\
\hline & $\boldsymbol{r}$ & $P$-value & $r$ & $P$-value & $r$ & $P$-value \\
\hline DEX-S total scores & & & 0.67 & $<0.0001$ & 0.48 & 0.01 \\
\hline DEX-I total scores & 0.50 & 0.01 & 0.40 & 0.03 & -0.07 & 0.70 \\
\hline BADS total scores & -0.15 & 0.42 & -0.29 & 0.12 & -0.11 & 0.56 \\
\hline EMC-S total scores & 0.67 & $<0.000$ I & & & 0.65 & 0.0001 \\
\hline EMC-I total scores & 0.40 & 0.03 & 0.48 & 0.01 & 0.14 & 0.46 \\
\hline RBMT total scores & 0.00 & 0.99 & 0.06 & 0.74 & 0.01 & 0.97 \\
\hline CFQ total scores & 0.48 & 0.01 & 0.65 & 0.0001 & & \\
\hline LNS scores & -0.22 & 0.24 & -0.07 & 0.71 & 0.11 & 0.55 \\
\hline $\begin{array}{l}\text { VMS forward } \\
\text { scores }\end{array}$ & 0.04 & 0.82 & -0.04 & 0.82 & -0.17 & 0.37 \\
\hline $\begin{array}{l}\text { VMS backward } \\
\text { scores }\end{array}$ & -0.16 & 0.39 & -0.18 & 0.33 & -0.01 & 0.95 \\
\hline CAS scores & 0.36 & 0.05 & 0.18 & 0.35 & 0.42 & 0.02 \\
\hline $\begin{array}{l}\text { MADRS total } \\
\text { scores }\end{array}$ & 0.08 & 0.67 & 0.07 & 0.74 & -0.02 & 0.90 \\
\hline HAM-A total scores & 0.29 & 0.12 & 0.31 & 0.09 & 0.16 & 0.40 \\
\hline BFI scores & 0.33 & 0.07 & 0.48 & 0.01 & 0.28 & 0.14 \\
\hline $\begin{array}{l}\text { FACT-B total } \\
\text { scores }\end{array}$ & -0.26 & 0.17 & -0.11 & 0.58 & -0.01 & 0.95 \\
\hline Hb levels & -0.03 & 0.87 & -0.21 & 0.26 & -0.05 & 0.78 \\
\hline TNF- $\alpha$ levels & 0.41 & 0.03 & 0.16 & 0.43 & 0.09 & 0.67 \\
\hline
\end{tabular}

Notes: We considered multiple corrections separately for primary (ie, CFQ, DEX-S, and EMC-S scores) and secondary outcome measures. Thus, a $P$-value is considered significant if it is less than $0.016(=0.05 / 3)$ and $0.0011(=0.05 /[3 \times 16-3])$ for the correlation analyses between primary outcomes and the other correlation analyses, respectively. Bold characters represent statistically significant results. Abbreviations: BADS, Behavioural Assessment of the Dysexecutive Syndrome; BFI, Brief Fatigue Inventory; CAS, Clinical Assessment for Spontaneity; CFQ, Cognitive Failures Questionnaire; DEX-I, independent-rater-rated Dysexecutive Questionnaire; DEX-S, self-rated Dysexecutive Questionnaire; EMC-I, independentrater-rated Everyday Memory Checklist; EMC-S, self-rated Everyday Memory Checklist; FACT-B, Functional Assessment of Cancer Therapy-Breast; HAM-A, Hamilton Anxiety Rating Scale; Hb, hemoglobin; LNS, Letter-Number Sequencing; MADRS, Montgomery-Åsberg Depression Rating Scale; RBMT, Rivermead Behavioural Memory Test; VMS, Visual Memory Span; TNF- $\alpha$, tumor necrosis factor- $\alpha$.

T0 to T1 between those who received radiotherapy $(n=13)$ and those who did not $(\mathrm{n}=17)$ (Tables $\mathrm{S} 1-\underline{\mathrm{S}} 3)$. The DEX-S scores at T0 and CFQ scores at T1 were higher in those who underwent hormone therapy during this study $(n=18)$ than those who did not $(n=9)$. No other significant differences were found in cognitive functions at $\mathrm{T} 0$ and at $\mathrm{T} 1$, and changes in cognitive functions from $\mathrm{T} 0$ to $\mathrm{T} 1$ between the groups (Tables S4- $\underline{\text { S6). }}$.

\section{Discussion}

To the best of our knowledge, this is the first prospective study to examine self-rated and family-rated cognitive functions encompassing multiple domains in patients with breast cancer following chemotherapy. We found that as early as within 4 weeks after chemotherapy, 20.0\% and
Table 4 Correlations among changes in self-rated cognitive functions and other clinicodemographic characteristics

\begin{tabular}{|c|c|c|c|c|c|c|}
\hline \multirow{3}{*}{$\begin{array}{l}\text { Changes from T0 } \\
\text { to TI }\end{array}$} & \multicolumn{6}{|c|}{ Changes from TI-T0 } \\
\hline & \multicolumn{2}{|c|}{ DEX-S } & \multicolumn{2}{|c|}{ EMC-S } & \multicolumn{2}{|l|}{ CFQ } \\
\hline & $r$ & $P$-value & $r$ & $P$-value & $r$ & $P$-value \\
\hline DEX-S total scores & & & 0.27 & 0.15 & -0.07 & 0.73 \\
\hline DEX-I total scores & 0.13 & 0.51 & -0.07 & 0.71 & -0.30 & 0.11 \\
\hline BADS total scores & 0.07 & 0.72 & -0.22 & 0.24 & -0.03 & 0.90 \\
\hline EMC-S total scores & 0.27 & 0.15 & & & 0.48 & 0.01 \\
\hline EMC-I total scores & -0.14 & 0.46 & -0.01 & 0.94 & -0.03 & 0.89 \\
\hline RBMT total scores & -0.06 & 0.75 & -0.13 & 0.51 & -0.10 & 0.59 \\
\hline CFQ total scores & -0.07 & 0.73 & 0.48 & 0.01 & & \\
\hline LNS scores & -0.18 & 0.34 & -0.06 & 0.77 & 0.04 & 0.85 \\
\hline VMS forward scores & -0.06 & 0.74 & -0.33 & 0.08 & -0.31 & 0.10 \\
\hline VMS backward scores & -0.31 & 0.10 & -0.13 & 0.49 & 0.31 & 0.10 \\
\hline CAS scores & $0.5 \mathrm{I}$ & 0.004 & 0.11 & 0.55 & 0.34 & 0.07 \\
\hline MADRS total scores & -0.05 & 0.79 & -0.12 & 0.55 & 0.26 & 0.17 \\
\hline HAM-A total scores & 0.06 & 0.75 & -0.14 & 0.47 & -0.04 & 0.82 \\
\hline BFI scores & 0.28 & 0.14 & 0.38 & 0.04 & 0.19 & 0.31 \\
\hline FACT-B total scores & -0.43 & 0.02 & -0.20 & 0.29 & -0.38 & 0.04 \\
\hline Hb levels & 0.04 & 0.83 & 0.04 & 0.82 & 0.16 & 0.40 \\
\hline TNF- $\alpha$ levels & -0.08 & 0.67 & -0.07 & 0.72 & 0.38 & 0.04 \\
\hline
\end{tabular}

Notes: We considered multiple corrections separately for primary (ie, CFQ, DEX-S, and EMC-S scores) and secondary outcome measures. Thus, a $P$-value is considered significant if it is less than $0.016(=0.05 / 3)$ and 0.0011 ( $(=0.05 /[3 \times 16-3])$ for the correlation analyses between primary outcomes and the other correlation analyses, respectively. Bold characters represent statistically significant results. Abbreviations: BADS, Behavioural Assessment of the Dysexecutive Syndrome; BFI, Brief Fatigue Inventory; CAS, Clinical Assessment for Spontaneity; CFQ, Cognitive Failures Questionnaire; DEX-I, independent-rater-rated Dysexecutive Questionnaire; DEX-S, self-rated Dysexecutive Questionnaire; EMC-I, independentrater-rated Everyday Memory Checklist; EMC-S, self-rated Everyday Memory Checklist; FACT-B, Functional Assessment of Cancer Therapy-Breast; HAM-A, Hamilton Anxiety Rating Scale; Hb, hemoglobin; LNS, Letter-Number Sequencing; MADRS, Montgomery-Åsberg Depression Rating Scale; RBMT, Rivermead Behavioural Memory Test; VMS, Visual Memory Span; TNF- $\alpha$, tumor necrosis factor- $\alpha$.

$6.7 \%$ presented with self-rated impairment of executive function and episodic memory, respectively. This is consistent with the prevalence rates reported by Ganz et al of $19.0 \%$ and $23.3 \%$ for subjective impairment in executive function and memory, respectively, 3 months after the primary breast cancer treatment $(n=189) .{ }^{31}$ On the other hand, in the present study, no participant showed impairment in self-reported attention 4 weeks after chemotherapy. This seems inconsistent with a previous study in which patients with breast cancer $(n=85)$ reported subjective worsening in attention following chemotherapy. ${ }^{12}$ This may be attributable to different sample characteristics and different batteries to quantify the trait in question. Furthermore, since the current study compared subjective attention between patients and normative data, the aforementioned discrepancy may be attributable to a limitation of our study regarding a lack of pre-chemotherapy cognitive results. 
At the 6-month follow-up, self-rated executive function improved, although this statistical significance failed to survive after multiple corrections. Among those who showed lower subjective executive function or episodic memory within 4 weeks after chemotherapy, each function was normalized in $50 \%$ and $100 \%$, respectively. On the other hand, no subject showed worsening in subjective cognitive functions. In support of this finding, Hermelink et al in a series of publications reported that patients with breast cancer $(n=101)$ showed deterioration in self-reported global cognition 5 months after chemotherapy and that they subsequently exhibited partial recovery. ${ }^{13,14}$ Further, Quesnel et al demonstrated that overall subjective cognition worsened between initiation and completion of chemotherapy, and returned to baseline levels 3 months after the completion of chemotherapy in this population $(n=41) .{ }^{9}$ Moreover, Biglia et al noted that patients with breast cancer $(n=40)$ did not report relevant cognitive problems before chemotherapy, and that no differences were found in subjective cognitive functions before and 6 months after chemotherapy. ${ }^{11}$ Lastly, Bender et al found no difference in overall subjective cognition between patients who received chemotherapy $(n=19)$ and those who did not $(n=19)$ before and 12 months after chemotherapy. ${ }^{15}$ Thus, these findings collectively suggest that subjective cognitive impairment may be transient after chemotherapy in most of patients with breast cancer. This notion is reinforced by the findings from the present study, which examined subjective (ie, self-rated and family-rated) cognitive domains such as executive function, episodic memory, and attention.

Furthermore, self-rated subjective cognitive functions (executive function, episodic memory, and attention) were associated with each other. Also, self-rated subjective cognitive functions (executive function and episodic memory) were linked to family-rated subjective cognitive functions within each domain and beyond the domains; only family-rated episodic memory was associated with self-rated episodic memory after correction for multiple comparisons. Thus, these findings suggest that patients with breast cancer and their families may share similar perspectives on cognitive functions following chemotherapy. On the other hand, self-rated executive function, episodic memory, and attention were related to TNF- $\alpha$, fatigue, and subjective motivation, respectively, within 4 weeks after chemotherapy. With regard to changes in self-rated cognitive functions at the 6-month follow-up, changes in self-rated executive function were related to changes in QOL, attention, and fatigue, those in episodic memory to those in self-rated short memory and QOL, and those in attention to those in TNF- $\alpha$. Six months after chemotherapy, self-rated executive function, episodic memory, and attention were associated with anxiety and QOL, anxiety and QOL, and anxiety, respectively. Although these correlations failed to survive correction for multiple comparisons, these findings are in line with both the lack of associations between subjective and objective cognitive functions and the presence of plausible relationships between subjective cognitive impairment and anxiety, fatigue, and QOL as have been identified in previous studies. ${ }^{8,30,32}$ In addition, it has been suggested that TNF- $\alpha$ is implicated in cognitive impairment induced by chemotherapy in this population. For example, Kesler et al reported that lower verbal memory performance was associated with higher cytokine levels and lower left hippocampal volume in patients with breast cancer following chemotherapy. ${ }^{16}$ Thus, taking our finding into account, TNF- $\alpha$ may serve as a relevant biomarker for subjective cognitive impairment in this population, which qualifies further investigations.

This study should be interpreted in light of its limitations. First, this study is a single-center study with a small sample size of Japanese patients with breast cancer. Thus, our findings are generalizable to other populations and necessitate further replication. Notably, a very few participants experienced subjective cognitive impairment compared with reported rates of $20 \%-80 \%$ in previous studies, ${ }^{30,33}$ which may be akin to type II errors caused by the limited sample size of this study. Second, quite a few participants underwent surgery, hormone therapy, or radiotherapy during this study, all of which have the potential to affect subjective cognitive functions, ${ }^{34}$ which is consistent with our finding that those who underwent hormone therapy during this study were associated with lower self-rated executive function at $\mathrm{T} 0$ and lower attention at $\mathrm{T} 1$ in comparison to those who did not. With regard to surgery, we could not examine the influence of this intervention on subjective cognitive functions since we did not examine them immediately before and after surgery in those who had received neoadjuvant chemotherapy and subsequently underwent surgery. Third, this study did not assess cognitive functions in patients prior to chemotherapy due to our consideration on the emotional state of patients immediately after receiving a diagnosis of breast cancer, although it has been noted that breast cancer patients show cognitive impairment even before chemotherapy. ${ }^{2}$ It would have been ideal to assess cognitive functions with neuropsychological tests before chemotherapy as the baseline data; the reason why we refrained from recruiting patients immediately after a diagnosis of breast cancer 
was our careful consideration on their emotional states in response to a highly stressful event. Moreover, we did not include control groups such as healthy subjects or patients with benign tumor. Instead, we compared between patients and normative data, leaving a possibility of practice effect although the tests were performed 6 months apart. Furthermore, since the normative data were obtained from published norms, the adequacy of the use of the normative data still remains unclear in relation to the specific study population. The strength of our study is the use of various batteries in an effort to better characterize subjective cognitive impairment, which is a serious clinical issue in light of a high prevalence of breast cancer across the globe.

In conclusion, this study indicated that subjective (ie, selfrated and family-rated) impairment in executive function, episodic memory, and attention may be transient and reversible after chemotherapy in patients with breast cancer. In light of the lack of associations between subjective and objective cognitive functions, both aspects of cognitive functions may need to be assessed in this patient population by themselves and their family members and medical staff throughout the illness course. Furthermore, the improved survivorship in patients with breast cancer leads to the increased importance to maintain QOL, which was found to be associated with subjective cognitive impairment in the present study. Thus, further research is warranted to elucidate the biological mechanisms (eg, inflammation) underlying cognitive impairments in this patient population, for which TNF- $\alpha$ may play a role of a relevant biomarker.

\section{Acknowledgment}

We would like to thank Dr T Maeda for insightful discussions on the study design and interpretation of the results.

\section{Disclosure}

Dr Ryosuke Kitahata has received Inokashira Hospital Research Grant within the past 3 years. Dr Shinichiro Nakajima has received fellowship grants from Canadian Institute of Health Research (CIHR), research support from Japan Society for the Promotion of Science, and manuscript fees or speaker's honoraria from Dainippon Sumitomo Pharma and Yoshitomi Yakuhin within the past 3 years. Dr Hiroyuki Uchida has received grants from Astellas Pharmaceutical, Eisai, Otsuka Pharmaceutical, Shionogi, Dainippon-Sumitomo Pharma, Eli Lilly, Mochida Pharmaceutical, Meiji-Seika Pharma, and Yoshitomi Yakuhin and speaker's honoraria from Otsuka Pharmaceutical, Eli Lilly, Shionogi, Pfizer, Yoshitomi Yakuhin, Dainippon-Sumitomo Pharma, Meiji-Seika
Pharma, MSD, and Janssen Pharmaceutical within the past 3 years. Dr Tetsu Hayashida has received grants and/or speaker's honoraria from Daiichi Sankyo, Eisai, Novartis Pharma, Takeda within the past 3 years. Dr Maiko Takahashi reports no conflicts of interest in this work. Dr Shintaro Nio has received speaker's honoraria from Astellas, Dainippon Sumitomo, Eisai, Eli Lilly, GlaxoSmithKline, Janssen Pharmaceutical, Mitsubishi Tanabe Pharma, Mochida Pharmaceutical, Otsuka Pharmaceutical, Pfizer, Shionogi, Takeda, and Yoshitomi Yakuhin within the past 3 years. Dr Jinichi Hirano has received grants from Eli Lilly and speaker's honoraria from Otsuka Pharmaceutical and Yoshitomi Yakuhin within the past 3 years. Mrs Maki Nagaoka has received Inokashira Hospital Research Grant within the past 3 years. Dr Takefumi Suzuki has received manuscript or speaker's honoraria from Astellas, Dainippon Sumitomo, Eli Lilly, Elsevier Japan, Janssen, Meiji Seika, Novartis, Otsuka, and Wiely Japan within the past 3 years. Dr Hiromitsu Jinno has received grants and/or speaker's honoraria from AstraZeneca, Pfizer, Chugai, Taiho, Novartis Pharma, and Kyowa Kirin within the past 3 years. Dr Yuko Kitagawa has received grants and/or speaker's honoraria from Astellas Pharmaceutical, Daiichi Sankyo, Dainippon-Sumitomo Pharma, Eisai, Eli Lilly, GlaxoSmithKline, Novartis Pharma, Pfizer, Shionogi, Takeda, Shionogi, and Otsuka Pharmaceutical within the past 3 years. Dr Masaru Mimura has received grants and/or speaker's honoraria from Asahi Kasei Pharma, Astellas Pharmaceutical, Daiichi Sankyo, Dainippon-Sumitomo Pharma, Eisai, Eli Lilly, GlaxoSmithKline, Janssen Pharmaceutical, Meiji-Seika Pharma, Mochida Pharmaceutical, MSD, Novartis Pharma, Otsuka Pharmaceutical, Pfizer, Shionogi, Takeda, Tanabe Mitsubishi Pharma, and Yoshitomi Yakuhin within the past 3 years.

\section{References}

1. Fallowfield L, Jenkins V. Psychosocial/survivorship issues in breast cancer: are we doing better? J Natl Cancer Inst. 2015;107(1):335.

2. Wefel JS, Kesler SR, Noll KR, Schagen SB. Clinical characteristics, pathophysiology, and management of noncentral nervous system cancer-related cognitive impairment in adults. CA Cancer J Clin. 2015; 65(2):123-138.

3. Ouimet LA, Stewart A, Collins B, Schindler D, Bielajew C. Measuring neuropsychological change following breast cancer treatment: an analysis of statistical models. J Clin Exp Neuropsychol. 2009;31(1):73-89.

4. Janelsins MC, Kesler SR, Ahles TA, Morrow GR. Prevalence, mechanisms, and management of cancer-related cognitive impairment. Int Rev Psychiatry. 2014;26(1):102-113.

5. Ahles TA, Saykin AJ, McDonald BC, et al. Cognitive function in breast cancer patients prior to adjuvant treatment. Breast Cancer Res Treat. 2008;110(1):143-152.

6. Jansen CE, Cooper BA, Dodd MJ, Miaskowski CA. A prospective longitudinal study of chemotherapy-induced cognitive changes in breast cancer patients. Support Care Cancer. 2011;19(10):1647-1656. 
7. Wefel JS, Saleeba AK, Buzdar AU, Meyers CA. Acute and late onset cognitive dysfunction associated with chemotherapy in women with breast cancer. Cancer. 2010;116(14):3348-3356.

8. Pullens MJ, De Vries J, Roukema JA. Subjective cognitive dysfunction in breast cancer patients: a systematic review. Psychooncology. 2010; 19(11):1127-1138.

9. Quesnel C, Savard J, Ivers H. Cognitive impairments associated with breast cancer treatments: results from a longitudinal study. Breast Cancer Res Treat. 2009;116(1):113-123.

10. Pullens MJ, De Vries J, Van Warmerdam LJ, Van De Wal MA, Roukema JA. Chemotherapy and cognitive complaints in women with breast cancer. Psychooncology. 2013;22(8):1783-1789.

11. Biglia N, Bounous VE, Malabaila A, et al. Objective and self-reported cognitive dysfunction in breast cancer women treated with chemotherapy: a prospective study. Eur J Cancer Care (Engl). 2012;21(4): 485-492.

12. Jenkins V, Shilling V, Deutsch G, et al. A 3-year prospective study of the effects of adjuvant treatments on cognition in women with early stage breast cancer. Br J Cancer. 2006;94(6):828-834.

13. Hermelink K. Acute and late onset cognitive dysfunction associated with chemotherapy in women with breast cancer. Cancer. 2010;117(5):1103; author reply 1103-1104.

14. Hermelink K, Untch M, Lux MP, et al. Cognitive function during neoadjuvant chemotherapy for breast cancer: results of a prospective, multicenter, longitudinal study. Cancer. 2007;109(9):1905-1913.

15. Bender CM, Sereika SM, Berga SL, et al. Cognitive impairment associated with adjuvant therapy in breast cancer. Psychooncology. 2006; 15(5):422-430.

16. Kesler S, Janelsins M, Koovakkattu D, et al. Reduced hippocampal volume and verbal memory performance associated with interleukin-6 and tumor necrosis factor-alpha levels in chemotherapy-treated breast cancer survivors. Brain Behav Immun. 2012;30 Suppl:S109-S116.

17. Vearncombe KJ, Rolfe M, Wright M, Pachana NA, Andrew B, Beadle G. Predictors of cognitive decline after chemotherapy in breast cancer patients. J Int Neuropsychol Soc. 2009;15(6):951-962.

18. Broadbent DE, Cooper PF, FitzGerald P, Parkes KR. The Cognitive Failures Questionnaire (CFQ) and its correlates. Br J Clin Psychol. 1982; 21(Pt 1):1-16.

19. Wilson BA, Alderman N, Burgess PW, Emslie H, Evans JJ. Behavioural Assessment of the Dysexecutive Syndrome (BADS). Bury St. Edmunds, UK: Thames Valley Test Company; 1996.
20. Wilson B, Cockburn J, Baddeley A, Hiorns R. The development and validation of a test battery for detecting and monitoring everyday memory problems. J Clin Exp Neuropsychol. 1989;11(6):855-870.

21. Hodgson KD, Hutchinson AD, Wilson CJ, Nettelbeck T. A metaanalysis of the effects of chemotherapy on cognition in patients with cancer. Cancer Treat Rev. 2013;39(3):297-304.

22. Wilson BA, Cockburn J, Baddeley AD. The Rivermead Behavioral Memory Test. Suffolk: Thames Valley; 1985.

23. Wechsler D. WAIS-R Manual. New York: The Psychological Corporation; 1981.

24. Wechsler D. Wechsler Memory Scale-Revised (Manual). San Antonio: The Psychological Corporation; 1987.

25. Montgomery SA, Asberg M. A new depression scale designed to be sensitive to change. Br J Psychiatry. 1979;134:382-389.

26. Hamilton M. The assessment of anxiety states by rating. $\mathrm{Br} \mathrm{J} \mathrm{Med}$ Psychol. 1959;32(1):50-55.

27. Mendoza TR, Wang XS, Cleeland CS, et al. The rapid assessment of fatigue severity in cancer patients: use of the Brief Fatigue Inventory. Cancer. 1999;85(5):1186-1196.

28. Kato M. The development and standardization of Clinical Assessment for Attention (CAT) and Clinical Assessment for Spontaneity (CAS). Higher Brain Funct Res. 2006;26(3):310-319.

29. Brady MJ, Cella DF, Mo F, et al. Reliability and validity of the Functional Assessment of Cancer Therapy-Breast quality-of-life instrument. J Clin Oncol. 1997;15(3):974-986.

30. Hutchinson AD, Hosking JR, Kichenadasse G, Mattiske JK, Wilson C. Objective and subjective cognitive impairment following chemotherapy for cancer: a systematic review. Cancer Treat Rev. 2012;38(7): 926-934.

31. Ganz PA, Kwan L, Castellon SA, et al. Cognitive complaints after breast cancer treatments: examining the relationship with neuropsychological test performance. J Natl Cancer Inst. 2013;105(11):791-801.

32. Breckenridge LM, Bruns GL, Todd BL, Feuerstein M. Cognitive limitations associated with tamoxifen and aromatase inhibitors in employed breast cancer survivors. Psychooncology. 2012;21(1):43-53.

33. Brueggemeier RW, Hackett JC, Diaz-Cruz ES. Aromatase inhibitors in the treatment of breast cancer. Endocr Rev. 2005;26(3):331-345.

34. Agrawal K, Onami S, Mortimer JE, Pal SK. Cognitive changes associated with endocrine therapy for breast cancer. Maturitas. 2010; 67(3):209-214.
Neuropsychiatric Disease and Treatment

\section{Publish your work in this journal}

Neuropsychiatric Disease and Treatment is an international, peerreviewed journal of clinical therapeutics and pharmacology focusing on concise rapid reporting of clinical or pre-clinical studies on a range of neuropsychiatric and neurological disorders. This journal is indexed on PubMed Central, the 'PsycINFO' database and CAS,

\section{Dovepress}

and is the official journal of The International Neuropsychiatric Association (INA). The manuscript management system is completely online and includes a very quick and fair peer-review system, which is all easy to use. Visit http://www.dovepress.com/testimonials.php to read real quotes from published authors. 